\title{
F responses studied with single fibre EMG in normal subjects and spastic patients ${ }^{1}$
}

\author{
H. H. SCH I L L E R A D E. ST T L B E R G \\ From the Department of Neurology, University Hospital, Zürich, Switzerland, \\ and the Department of Clinical Neurophysiology, Academic Hospital, Uppsala, Sweden
}

S U M MARY Recurrent responses of single human motor neurones after antidromic activation, the so-called $\mathrm{F}$ response, have been studied in the abductor digiti minimi muscle in normal subjects and in spastic patients with the single fibre EMG technique. The $F$ response occurs rarely and in groups. Half of all motor neurones did not produce any $F$ response within an arbitrarily chosen time interval of 200 successive stimuli at 1 and $2 \mathrm{~Hz}$. Spastic patients had as many silent neurones as the normal subjects but the responding neurones more frequently produced $F$ responses $(P<0.001)$. When a neurone has few responses at rest the frequency increases under activation of the contralateral or ipsilateral muscle $(P<0.05)$. When there are many responses in the relaxed state there is a decrease $(P<0.001)$ under activation. The behaviour of an individual neurone is consistent in different activation periods.

After stimulation of a peripheral nerve, a late response may be recorded with surface electrodes over the corresponding muscle at a latency of about 20 to $30 \mathrm{~ms}$ in the upper extremities (Conrad et al., 1975) and 40 to $60 \mathrm{~ms}$ in the foot muscles, depending on the length of the extremity. This late response consists of $H$ reflexes and $F$ waves. These two components behave in different ways on changing the strength of electrical stimulation. The $\mathrm{H}$ reflex has its maximal amplitude with low stimulation strength, and decreases with further increase of stimulation strength. The $F$ wave increases in amplitude with increasing stimulation strength. The $\mathbf{H}$ reflex is more pronounced in the lower extremity, and the $F$ response in the upper extremity. With surface electrodes $\mathbf{H}$ and $F$ responses cannot be distinguished clearly, since both might be present throughout the whole range of stimulation (Hagbarth, 1962). Single fibre electromyography (SFEMG) allows clear distinction between these two components (Trontelj, 1973a) because the $H$ reflex is only rarely preceded by an $M$ response (Trontelj, in preparation), and has a jitter of about $150 \mu \mathrm{s}$. In contrast, the $\mathbf{F}$

\footnotetext{
Address for reprint requests: Dr H. H. Schiller, Department of Neurology, Kantonspital, CH 8091 Zürich, Switzerland.

${ }^{1}$ This work was supported by the Swedish Medical Research Council (ES, Grant No. B-135) and the Schweizerische Stiftung für BiologischMedizinische Stipendien (HHS), and the European Training Programme in Brain and Behaviour Research (Twinning grant).

Accepted 19 August 1977
}

response is always preceded by an identical $M$ response and has a jitter only slightly greater than that of the $M$ response, usually less than $50 \mu \mathrm{s}$ (Fig. 1) (Trontelj, 1973a; Schiller and Stålberg, 1977).

The $F$ wave recorded by surface electrodes is known to be much smaller than the direct compound $M$ response; its shape and latency are variable, and it does not follow each $M$ response (Magladery and McDougal, 1950).

Since the first description of the $F$ wave by Magladery and McDougal (1950) there has been much controversy about its origin and significance. Magladery and McDougal (1950) assumed that it was a spinal reflex response transmitted by slowly conducting afferent nerve fibres. Dawson and Merton (1956) were the first to suggest that the $F$ wave was produced by motor neurone discharge after antidromic activation by centripetal volleys. Thorne (1965) lent additional support to the antidromic genesis of this late response in an investigation with bipolar electrodes which allowed more selective recordings of individual motor unit responses than the ordinary concentric EMG electrode or surface electrodes. He showed that an $F$ wave occurs only after the direct $M$ response from the same motor unit. In spastic patients he found a late polyphasic component, the nature of which was uncertain. Gassel and Wiesendanger (1965) produced experimental evidence in cats 
H reflex

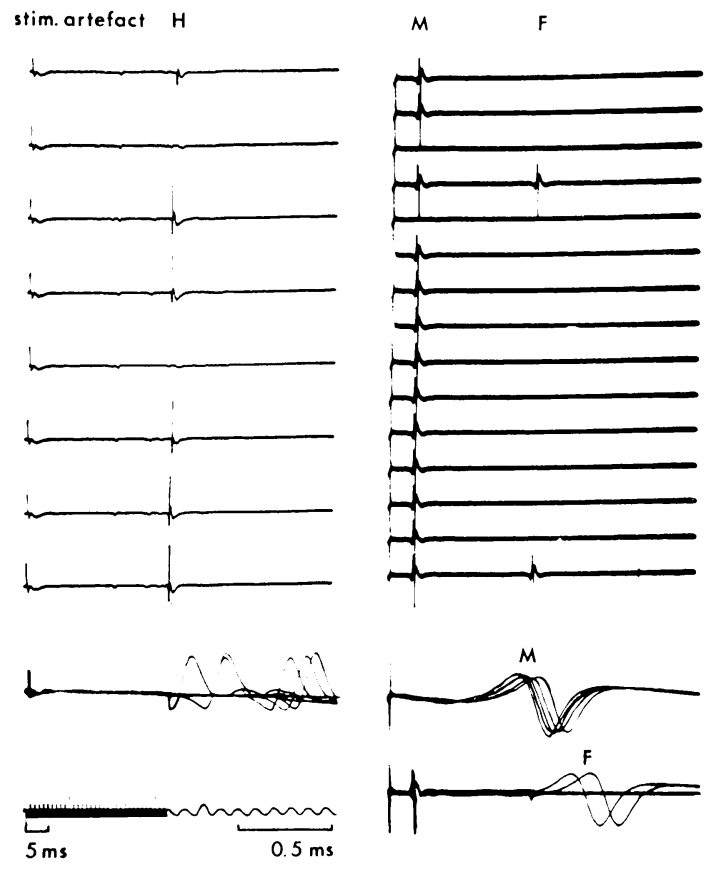

Fig. 1 Single fibre EMG recordings of $H$ reflex and of an $F$ response. The $H$ reflex is not preceded by an $M$ response and its latency variability, the jitter. is of the order of several hundreds of microseconds. The $F$ response is always preceded by an $M$ response and its jitter is of the same order as that of the $M$ response. In the lower part the oscilloscope sweeps are superimposed and partially expanded to identify the shape of the component examined.

that the late response, as obtained from small limb muscles on nerve stimulation, consisted of two components-an early response not abolished by deafferentation and, therefore, representing a motor neurone discharge on antidromic activation, and a late polyphasic response, which was abolished by deafferentation and which they interpreted as a polysynaptic reflex. McLeod and Wray (1966) concluded from studies in the baboon that the main component of the $F$ wave was a recurrent response resulting from antidromic motor neurone activation. The recurrent nature of the $F$ wave on antidromic activation has since been confirmed and further studied by Tronteli (1973a) and Tronielj and Trontelj (1973) using the SFEMG technique. Quantitative data about the occurrence of the $F$ wave are, however, lacking.

The purpose of the present investigation was to obtain information about the natural occurrence and history of the $\mathrm{F}$ response of single motor neurones as recorded by SFEMG in normal subjects and in spastic patients.

\section{Subjects and method}

Twenty-eight experiments were performed in 18 healthy subjects, 12 men and six women, aged 14 to 70 years. $F$ waves of 145 different motor units were analysed. The subjects reclined comfortably on a couch and care was taken to ensure that they were relaxed. In six spastic patients, $F$ waves of a total of 60 motor units were examined. Three of these patients had spasticity of their right arm due to a thrombosis of their left middle cerebral artery, two patients had spasticity after operation for a brain tumour, and one suffered from infantile cerebral palsy. All patients had increased muscle tone and brisk tendon jerks on the affected side but were otherwise in good health and were able to walk. The results from the spastic arm (40 action potentials) were compared with the findings in the non-spastic arm (20 potentials).

The ulnar nerve was electrically stimulated re- $\mathbb{D}$ petitively at the elbow or wrist with surface bipolar electrodes (Disa 13K62). In four patients stimulation was done with insulated tungsten electrodes with a bare tip of $3 \mathrm{~mm}$. The stimulation unit was a Grass S88, Disa 14E12, or Disa 15E05. The stimulation rate was varied in different experiso ments from 0.5 to $5 \mathrm{~Hz}$. Pulse width was 0.05 or $0.1 \mathrm{~ms}$. Stimulation strength was adjusted to produce a suprathreshold $M$ response (Stålberg et al., 1973) for the motor unit under study, up to $3 \mathrm{~V}$ (needle electrodes) and $150 \mathrm{~V}-10 \mathrm{~mA}$ (surface electrodes). The $M$ response sometimes became complex or disappeared, probably due to changes in the electrode or skin resistance or both. This was checked in three control experiments by measuring the galvanic skin resistance. This effect was avoided by slight adjustments of the stimulation strength.

Muscle action potentials were recorded from the abductor digiti minimi muscle (ADM) with a single fibre EMG electrode (Medelec SF25). Further details of the SFEMG method are given by Stålberg and Ekstedt (1973).

An electrode position was sought where an $M$ response fulfilling the criteria of a single muscle fibre action potential (Eksedt, 1964) was obtained on stimulation of the ulnar nerve. Complex action potentials were rejected for that part of the study where the criteria for $\mathrm{H}$ and $\mathrm{F}$ waves were specially investigated, as such potentials may originate from muscle fibres belonging to several motor 
units. Responses composed from more than one muscle fibre were accepted when the $M$ wave responded in an 'all-or-none' manner to changes in stimulation strength. In some investigations, complex recordings were accepted when they contained distinct single muscle fibre action potentials. However, the late response had to be of the same shape as the single fibre component in the preceding $M$ response (Fig. 2). In some experiments information could be obtained about two motor neurones of different thresholds.

A late response was identified and classified as an $F$ response (Trontelj, 1973a) when it had the same shape as the preceding direct $M$ response, and when the latency variability, the jitter, was only slightly greater than the corresponding $M$ wave jitter. The latency of the $F$ wave had to fall within the known range of 20 to $30 \mathrm{~ms}$, depending on the length of the arm (Conrad et al., 1975). Potentials at shorter latencies were rejected even when the shape of the potential was constant and its latency variability was small, as this may occur in axon reflexes (Fullerton and Gilliatt, 1965; Stålberg et al., 1973). The potentials were stored on analogue tape. The jitter of the $M$ and $F$ waves was computed off-line by a PDP 11/40 computer using a Hewlett-Packard HP 5326 B time interval counter. The jitter was expressed as mean consecutive difference (MCD) of fifty $M$ and five $F$ responses respectively. Sequential and nonsequential time interval histograms of the occurrence of the $F$ wave were also computed.

If different motor units were examined in one subject, the position of both the stimulating electrode and the recording electrode were changed, in order to increase the probability of recording different motor units. The same recording position could sometimes be used to study two neurones if they had different stimulation thresholds.

The $\mathrm{F}$ waves were investigated both at rest and during activation. The investigations at rest were usually long-term experiments, in which recordings were made from the same motor unit for between 25 and 40 minutes. Activation tests included contraction of the contralateral ADM, slight contraction of the ipsilateral ADM, contraction of the gastrocnemius, and hyperventilation. In one experiment vibration of amplitude $1.5 \mathrm{~mm}$ and frequency $100 \mathrm{~Hz}$ was applied at different sites on the ipsilateral arm.

Neither long-term experiments nor different test manoeuvres could be performed systematically in the spastic patients, because of difficulties associated with background contraction in the spastic arm.
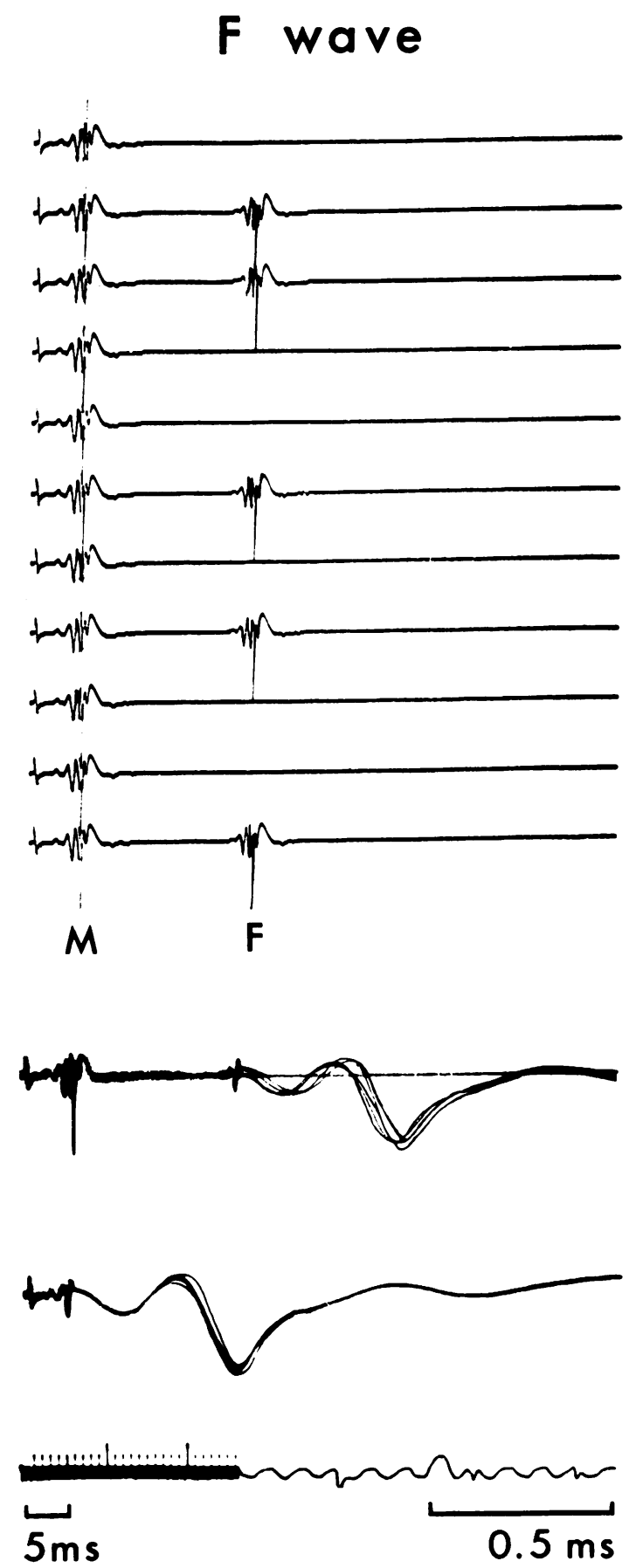

Fig. 2 Complex recording of action potentials belonging to the same motor unit (time locked to each other and responding in an all-or-none manner). The $F$ response has the same shape as the preceding $M$ response. 


\section{Results}

F RESPONSE JITTER

The jitter values in the normal subjects were below $70 \mu$ s for all motor units. Only three action potentials showed a jitter between 50 and $70 \mu$ s and in two recordings this higher value was due to threshold stimulation and was associated with a correspondingly higher jitter of the $\mathrm{M}$ response (Stålberg et al., 1973). The mean jitter of the F responses (excluding the two potentials mentioned above) was $40 \pm 10 \mu \mathrm{s}(\mathrm{n}=17)$. The jitter of the M response was always below $40 \mu$ s, typically 10 to $30 \mu \mathrm{s}$.

The $\mathrm{F}$ wave in the spastic patients had a slightly higher jitter (Fig. 3) $45 \pm 5 \mu \mathrm{S}(\mathrm{n}=14)$. The difference between these two mean values was not significant $(\mathrm{P}=0.1)$.

\section{F Jitter}
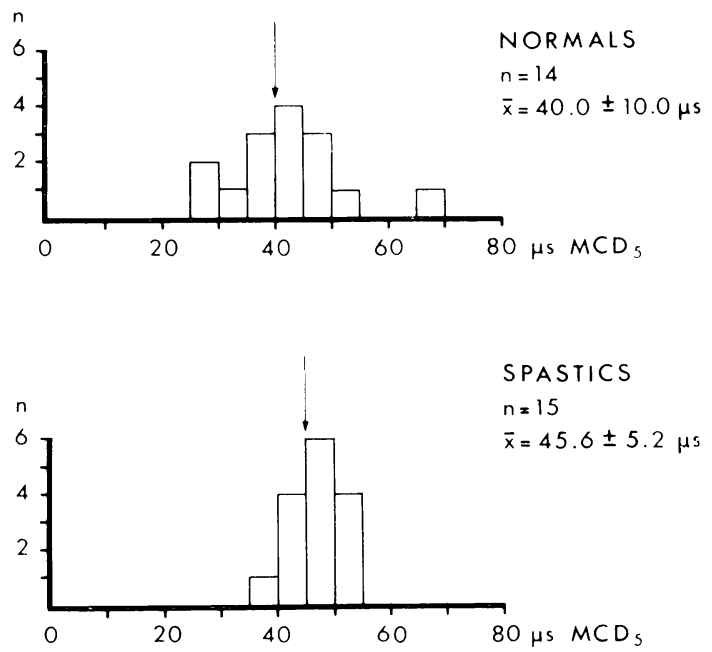

Fig. 3 Histogram of the F response jitter, expressed as mean consecutive difference of five responses, upper diagram normal subjects, lower diagram spastic

patients. There is a slight but not significant difference in mean values.

STIMULATION FREQUENCY

In four experiments the influence of the stimulus frequency on the occurrence of the $F$ response was tested. Stimulation was performed at 0.5 , 1 , and $5 \mathrm{~Hz}$. The stimulation period contained about $800 \mathrm{M}$ responses at each frequency tested in each experiment. The mean frequency of $F$ responses for 800 stimuli was $2.07 \pm 1.27$ at $0.5 \mathrm{~Hz}, 1.85 \pm$ 0.86 at $1 \mathrm{~Hz}$, and $1.91 \pm 0.4$ at $5 \mathrm{~Hz}$. Since no significant difference was noted at the different stimulation frequencies, we chose $1 \mathrm{~Hz}$ or $2 \mathrm{~Hz}$ stimulation for further experiments, rejecting $5 \mathrm{~Hz}$ because of the increased risk of displacement of the electrode with fast muscle twitches.

\section{OCCURRENCE OF F RESPONSES IN RELAXATION}

In order to obtain information about the natural behaviour of the $F$ response, 11 long-term experiments were done, in which the same potential was recorded for periods of 20 to 45 minutes. All investigations showed that the $F$ responses were unevenly distributed in time. Two examples are shown in Fig. 4. There were periods equivalent to $600 \mathrm{M}$ waves $(=10$ minutes at $1 \mathrm{~Hz}$ stimulation rate) during which no $F$ responses were seen. The $F$ responses occurred in groups. The non-sequential time interval histogram between two successive $F$ responses showed a highly skewed distribution (Fig. 5), in contrast to the Gaussian distribution to be expected with a random event. From these experiments it is seen that the $\mathrm{F}$ responses occur rarely and do not follow each $M$ response. However, we noted frequently that $F$ responses could be recorded with successive stimuli. To characterise a motor neurone in terms of $F$ responses, a long observation period is required. In order to estimate the number of stimuli necessary to characterise a mean frequency of $F$ responses in one motor neurone the following analysis was performed: the mean number of $F$ responses occurring in different sized groups of consecutive $M$ responses was compared to the mean frequency of the $F$ response for the whole experiment. For each group size the number of groups showing an $\mathrm{F}$ response frequency differing by more than $\pm 100 \%$ from the total mean was calculated. With a group size of 50 stimuli, between 30 and $50 \%$ of the groups had unrepresentative results. Groups with 100 stimuli had unrepresentative results in 20 to $30 \%$, and with 200 stimuli $10 \%$ of the groups showed unrepresentative results. With 500 stimuli all groups showed values within $\pm 100 \%$ of the mean of the whole experiment. To optimise both time for the experiment and accuracy of the results, we considered that a group of 200 stimuli at $1-2 \mathrm{~Hz}$ was a convenient number to characterise the occurrence of the $F$ response.

FREQUENCY OF OCCURRENCE

When the occurrence of the $F$ response in different motor units was calculated from investigations with 200 stimuli, we found that $55 \%$ of all motor neurones $(n=75)$ tested did not produce any $F$ response during that period (Fig. 6a), 35\% showed 


\section{$F$ frequency in relaxation}

A

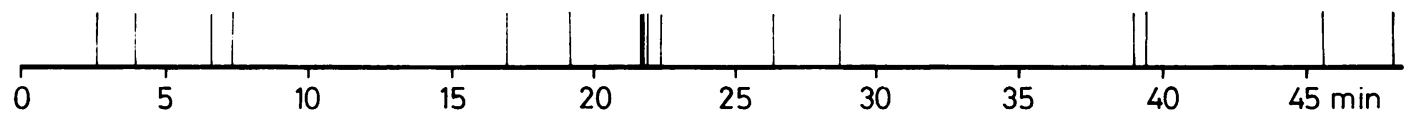

$B$

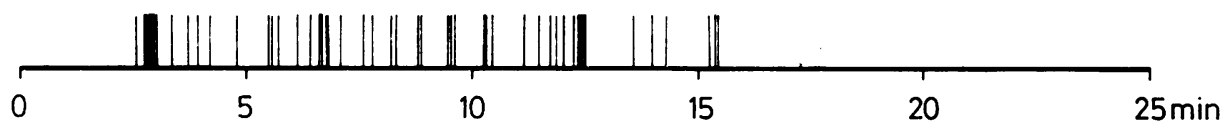

Fig. 4 Occurrence of the $F$ response in two long-term experiments, $A$ and $B$. Abscissae $=$ time axis. Each line represents one $F$ response. Note uneven distribution of $F$ responses.
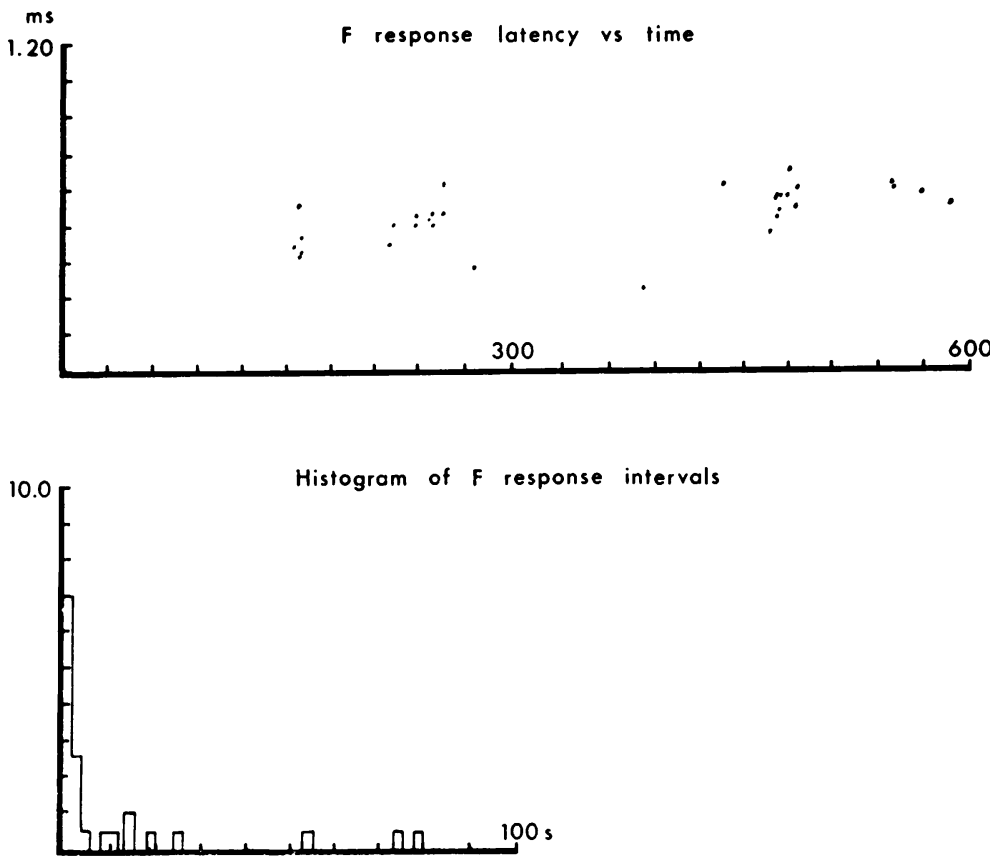

Fig. 5 Upper part: computer printout of the $F$ response latency (ordinate) versus time (abscissae). The variability of the latency represents the jitter. Lower part: histogram of the intervals between two consecutive $F$ responses. Note the skew distribution. one to five $\mathrm{F}$ responses, $4 \%$ showed five to 10 , and $2 \%$ between 10 and 15 . There was no neurone with more than $15 \mathrm{~F}$ responses per $200 \mathrm{M}$ responses (Fig. 6A).

\section{ACTIVATION}

The discharge characteristics of motor neurones in terms of $F$ response frequency changed during activation produced by slight contraction of the contralateral ADM or contraction of the ipsilateral ADM. Two examples are shown in Fig. 7. In Fig. 7A the mean number of $F$ responses increased from 1.82 to $30.4 \pm 18.5$ per $200 \mathrm{M}$ responses during activation. The difference between the relaxed and the activated period was signifi- cant $(P<0.05)$. The number of $F$ responses on the contrary decreased in the experiment shown in Fig. 7B from 32 at rest to two per $200 \mathrm{M}$ responses during activation $(\mathrm{P}<0.001)$. In five recordings with few or no responses at rest, there was an increase in the number of $F$ responses during activation of the contralateral or ipsilateral ADM muscle (Fig. 8A). In five recordings with a high number of $F$ responses at rest there was a decrease in, or even a total inhibition of, the number of $\mathrm{F}$ responses during activation (Fig. $8 \mathrm{C}$ ). In Fig. 8B three typical neurones are shown which did not change their discharge characteristics significantly during activation. In all neurones at least one $F$ response could be elicited either during rest or 


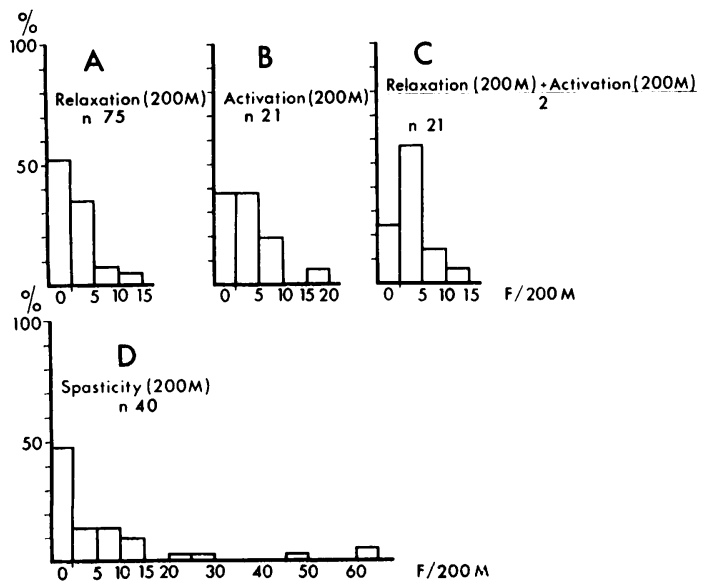

Fig. 6 Histogram of the frequency of $F$ responses per 200 stimuli (and $200 \mathrm{M}$ responses), $A$ in relaxation, $B$ under activation of ipsilateral or contralateral muscle, $C$ in pooled material (relaxation + activation divided by two), and $D$ in spastic patients. Percentage of 0 responses decreases when both activation and relaxation periods are considered. In spasticity there are higher numbers of $F$ responses than in normal subjects.

during activation. If the mean value of all relaxed periods is compared with the mean value of all activation periods in the three categories of neurones, there was for the increasing neurones a change in $F$ response incidence from 0.6 to 8.25 $(n=16, p<0.05)$. For the diminishing neurones there was a decrease from 7.3 to $1.13(n=11$, $\mathbf{p}<0.001$ ). The consistency of this behaviour was tested in 12 neurones by repeating the activation manoeuvres up to six times. There was no neurone which, for example, showed an increase under one activation period but a decrease in the next one. Five neurones did not change the $F$ response incidence during rest and activation. Their $F$ response incidence was 2.4 and 2.3 during rest and at activation respectively.

In two experiments with hyperventilation an increase could be observed, but simultaneous EMG activity was also recorded from the muscle studied. Contraction of the quadriceps muscle (two experiments) did not produce any change in the occurrence of the $F$ response. The manoeuvres of activation tests such as coactivation of the ipsiand contralateral ADM, or contraction of the quadriceps, could not be tested in all motor units as the recording electrode was easily displaced by the manoeuvres. In such a case this test had to be discarded because the potentials no longer fulfilled the criterion of constant shape. Hyperventilation and vibration proved even more difficult, so no systematic data could be obtained with these tests. In the whole material where the occurrence of $F$ responses was compared in relaxation and during activation, the frequency of $F$ responses per $200 \mathrm{M}$ responses was approximately the same (Fig. 6A, B). When the number of $F$ responses was pooled from both relaxation and activation periods, there was a lower number of neurones with no discharges, $23 \%$ compared to $38 \%$ during activation and 53 during relaxation (Fig. 6C).

\section{SPASTICITY}

In six spastic patients the $F$ response distribution? and occurrence was investigated and compared with both the non-spastic side and the healthy subjects. The number of late responses in the spastic group was very high. With the criteria mentioned above it was seen that most of these were $\mathrm{H}$ reflexes, not dealt with in this paper. $\mathrm{H}$

\section{Change in $F$ frequency with activation}

A

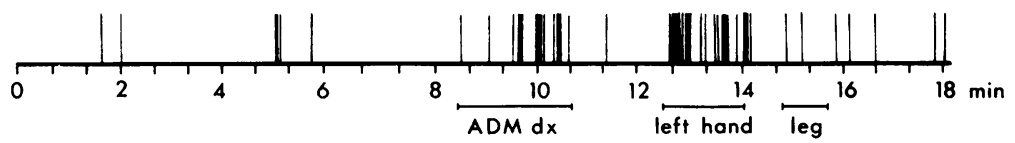

B

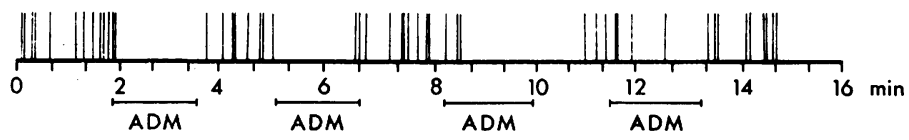

Fig. 7 Two long-term experiments, $A$ and $B$, with a pronounced change in occurrence of the $F$ response under activation. In $A$ there was a small number of $F$ responses in relaxation, consistently increasing under activation of the ipsilateral and contralateral muscles. In B there was a high number in relaxation, decreasing consistently under activation of the muscle being recorded. 
1

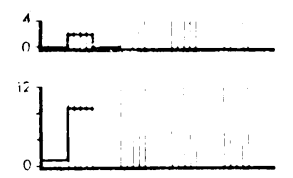

Rest

Activity

111
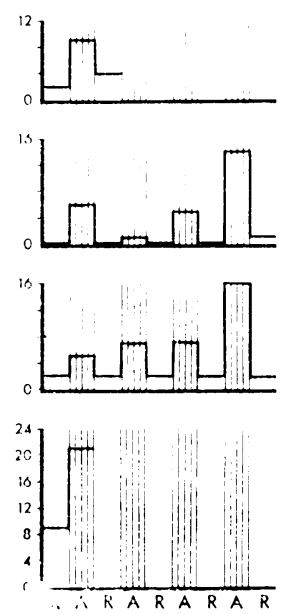

\section{II}
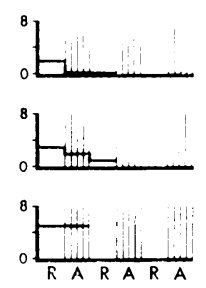
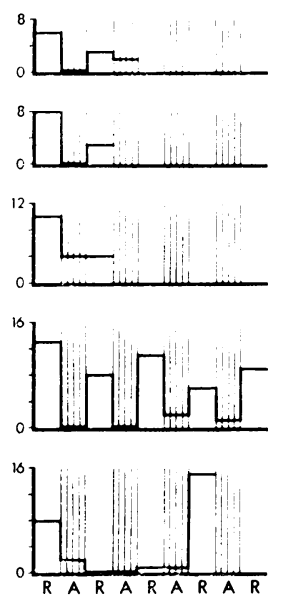

IV

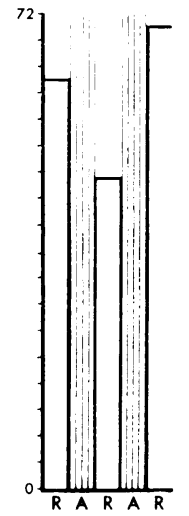

Fig. 8 Summary of the changes in $F$ response frequency at rest $(R$. open columns) and under activation ( $A$, lined columns)-I neurones with an increasing frequency. II neurones without significant difference, III neurones with a decreasing frequency, and $I V$ one typical case of spasticity with a pronounced decrease under activation. Note the consistency of the responses within the same neurone. reflexes were never seen in ADM in normal subjects.

The occurrence of $F$ responses was uneven, similar to that in normal subjects (Fig. 4). As mentioned in the section on the $F$ response jitter, there was no significant difference from the normal group (Fig. 3). The $F$ response frequency on the spastic side was higher than that of the nonspastic side, and higher than that in the healthy subjects. On the spastic side $47 \%$ of the neurones did not show any $F$ responses during 200 stimuli. A similar frequency was found in normal subjects (53\% at rest and $38 \%$ in the activated state) (Fig. $6 \mathrm{~B}, \mathrm{D})$. The number of discharges of the responding neurones in the spastic patients, however, was increased. Fifteen per cent of the neurones had up to five $\mathrm{F}$ responses per $200 \mathrm{M}$ responses, $15 \%$ up to 10 , and $10 \%$ up to 15 responses. Fifteen per cent of the neurones showed over $20 \mathrm{~F}$ responses per $200 \mathrm{M}$, the highest value being 66 . The average response on the spastic side was one $F$ response in $20 \mathrm{M}$ responses $(n=20)$ and on the non-spastic side one $\mathrm{F}$ response in $66 \mathrm{M}$ responses. In the normal material there was one $F$ response in $118 \mathrm{M}$ responses. The differences from the nonspastic side and from the control group were both significant $(\mathrm{P}<0.001)$. The differences between the non-spastic side and the normal group were significant $(\mathbf{P}<0.001)$. In one case it was seen that the number of $F$ responses decreased from 66 during relaxation to none during activation (Fig. 8D).

\section{Discussion}

The $F$ wave is an intriguing and interesting artefact in neurophysiology, but it can provide information about the state of the lower motor neurone. The $F$ response, as examined by SFEMG. is always preceded by its corresponding $M$ response, although in complex $M$ responses the shape may not be distinguished clearly. In the latter case, $F$ responses were not accepted in this study. The shape of the $F$ wave has to be constant in successive $F$ discharges, whether it be a single fibre potential or a complex potential. These prerequisites mean that the $F$ response is transmitted by the same axon as the one evoking the direct $M$ response. The latency variability, the 'jitter', of the $F$ response, is small and only slightly greater than the corresponding $\mathbf{M}$ jitter (Stålberg et al., 1973; Trontelj, 1973a). Such a small jitter which is only 10 to $30 \mu \mathrm{s}$ greater than the corresponding $M$ jitter, excludes the possibility of synaptic transmission being involved in the aetiology of the $\mathrm{F}$ response. The $\mathrm{H}$ reflex, which is considered to be a monosynaptic reflex (Hoffmann, 1922), has a latency variability of about $150 \mu \mathrm{S}$ (Trontelj, 1973b). 
The neurophysiological mechanisms of recurrent discharge of motor neurones after antidromic activation have been established and reviewed by Renshaw (1941) and Eccles (1955). Within the pathway of the antidromic action potential there are three locations which are critical for further propagation (Fig. 9). These three locations are the proximal part of the myelinated axon (M), the axonal hillock (MN), and the soma-dendritic membrane (SD). When the antidromic action potential is blocked at any of these sites, intracellular recordings show the two different potentials transmitted electronically from the respective site. Only when a spike potential of the somadendritic membrane occurs (SD spike), may the action potential be transmitted orthodromically (recurrent response), and this occurs only if the membrane on the NM and in the M part can be depolarised again. This is usually the case because the membrane potential at these sites is brief and has only minimal after-hyperpolarisation. Usually invasion of the soma occurs rarely as reflected by the low number of $\mathrm{F}$ responses. Blocking of the antidromic potential usually occurs at the axonal hillock (Eccles, 1955) due to the change in membrane surface at the transition between axon and soma. When the soma-dendritic membrane is already slightly depolarised, the invasion of an antidromic action potential is facilitated, causing an SD spike. This will normally give rise to a recurrent response. However, if the soma-membrane is already excessively depolarised, the soma-dendritic spike occurs earlier, and the recurrent discharge is not elicited because the membrane at the axonal hillock is not yet repolarised.

Thus a recurrent response is limited to a narrow time period, limited on one hand by the latest possible SD spike and on the other hand by the repolarisation of the axonal hillock. This time range is small as reflected in the pure $F$ response jitter-that is, the measured jitter minus the $M$ jitter, a value of 10 to $30 \mu \mathrm{s}$. The above-mentioned change in frequency of $\mathrm{F}$ response due to preexisting depolarisation can occur due to spatial and temporal summation of EPSPs on activation of the ipsilateral or contralateral muscle. In neurones with a low number of $F$ responses there is probably an increase in the number of SD spikes on antidromic activation. In neurones with an initially high number of $\mathrm{F}$ responses activation caused a reduction, probably as an effect of blocking too early SD spikes at the axonal hillock. In spasticity, where pre-existing depolarisation of the somatic membrane can be assumed, the number of $\mathrm{F}$ responses was high and additional activation had a negative effect. We did not find other definite differences between a spastic motor neurone and a normal motor neurone under activation.

Moreover, the change in $F$ response frequency could be observed only when activation concerned the same spinal segment. The frequency of $F$ response in one neurone is generally low, from 0 to 15 per 200 stimuli. However, in $\vec{c}$ longer experiments all motor neurones showeck occasional $\mathrm{F}$ responses. This finding is in contrasto to the situation in the orbicularis oculi musclee where $F$ responses could not be obtained fromo some motor neurones (Trontelj, in preparation) Although we did not find any $\mathrm{H}$ responses in normal subjects in our experimental situation, this possibility is not excluded. Typical $\mathrm{H}$ responses were found by Trontelj (1973a) in abductor digiti

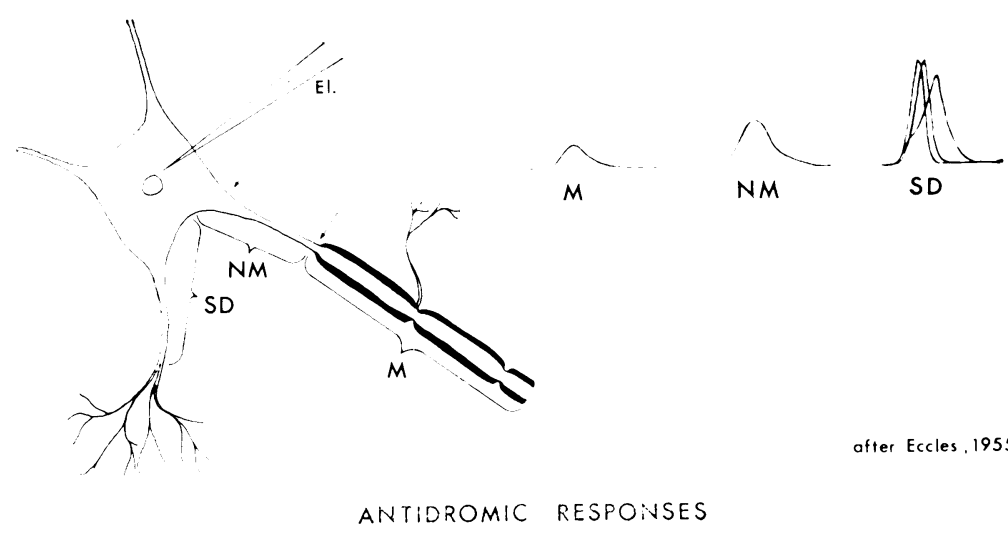

Fig. 9 Schematic drawing after Eccles (1955) showing potentials recorded with an intracellular electrode after antidromic activation. When the antidromic action potential is blocked at $M$, a so-called $M$ spike is recorded, when it is blocked at the axonal hillock, an NM spike is recorded. These potentials represent electrotonic conduction of membrane potential changes. When the antidromic action potential invades the soma an $S D$ spike is recorded. Note the different latencies of the latter due to the trigger point at different slopes of the preceding electrotonically conducted NM potential. 
minimi, and they might be recruited more frequently during post-tetanic potentiation or voluntary activation or both, as reported by Hagbarth (1962). The reason why we did not see this $H$ reflex during voluntary contraction may be that it was difficult to keep the recording selective at higher degrees of voluntary contraction.

While stimulating the alpha-axon it is evident that the current applied depolarises many axons with lower threshold, especially Ia afferent fibres. These conduct the action potential orthodromically to the spinal cord where they are linked mono-and/or polysynaptically to the motor neurone. There are excitatory and inhibitory potentials summating on each motor neurone which are transmitted by the afferent axon through the stimulation applied. In addition there are IPSPs transmitted by Renshaw cells which are excited antidromically (Renshaw, 1941; Eccles, 1955). The invasion of the antidromic action potential must thus be explained on the background of these concomitant events.

The overall incidence of the $F$ responses seen in the present study of single motor units is probably representative for the motor neurone pool of ADM since the surface $F$ wave is of the order of $1 \%$ of the $\mathbf{M}$ wave amplitude. It should be noted, that in this investigation there is a bias against larger neurones (Hennemann et al., 1965) as the larger axons have a lower threshold for stimulation.

The low amplitude of the $F$ wave studied by surface electrodes could be due either to a small number of neurones responding frequently or to a large part of the motor neurone pool responding infrequently. Our studies support the latter explanation. The discrepancy between the small jitter in the SF recordings and the variable latency of the surface recorded $F$ wave is probably, then, due to the fact that consecutive $F$ responses seen with surface electrodes represent responses from different motor neurones.

\section{References}

Conrad. B., Achoff, J. C., and Fischler, M. (1975). Der cliagnostische Wert der F-Wellen Latenz. Journal of Neurology, 210, 151-160.

Dawson, G. D., and Merton, P. A. (1956). 'Recurrent' discharges from motor neurones. Abstract. 20th International Congress of Physiology, Bruxelles, 221-222.

Eccles. J. C. (1955). The central action of antidromic impulses in motor nerve fibres. Pflügers Archiv für die Gesamte Physiologie des Menschen und der Tiere, 260, 385-415.
Ekstedt, J. (1964). Human single fibre action potential. Acta Physiologica Scandinavica, 61, Suppl. 226, $1-96$.

Fullerton, P. M., and Gilliatt, R. W. (1965). Axon reflexes in human motor nerve fibres. Journal of Neurology, Neurosurgery, and Psychiatry, 28, 1-11.

Gassel, M. M., and Wiesendanger, M. (1965). Recurrent and reflex discharges in plantar muscles of the cat. Acta Physiologica Scandinavica, 65, 138-142.

Hagbarth, K. E. (1962). Post-tetanic potentiation of the myotatic reflexes in man. Journal of Neurology, Neurosurgery, and Psychiatry, 25, 1-12.

Hennemann, E., Somjen. G., and Carpenter, D. O. (1965). Functional significance of cell size in spinal motor neurones. Journal of Neurophysiology, 28, $560-580$.

Hoffmann, P. (1922). Untersuchungen über die Eigenreflexe menschlicher Muskeln. Springer: Berlin.

Magladery, J. W., and McDougal, D. B. (1950). Electrophysiological studies of nerve and reflex activity in normal man. I. Identification of certain reflexes in the electromyogram and the conduction velocity of peripheral nerve fibers. Bulletin of the Johns Hopkins Hospital, 86, 265-290.

McLeod, J. G., and Wray, S. H. (1966). An experimental study of the $\mathrm{F}$ wave in the baboon. Journal of Neurology, Neurosurgery, and Psychiatry, 29, 196-200.

Renshaw, B. (1941). Influence of discharge of motor neurones upon excitation of neighboring motor neurones. Journal of Neurophysiology, 4, 167-183.

Schiller, H. H., and Stålberg, E. (1977). The F wave studied by single fiber electromyography. Electroencephalography and Clinical Neurophysiology, 42, 130.

Stålberg, E., and Ekstedt, J. (1973). Single fibre EMG and the microphysiology of the motor unit in normal diseased human muscle. In New Developments in Electromyography and Clinical Neurophysiology. Edited by J. E. Desmedt, Volume 1, pp. 113-129. Karger: Basel.

Stålberg, E., Trontelj, J. V., Trontelj, M. (1973). The jitter of single human muscle fibre responses to stimulation of the motor axon. (Abstract). Electroencephalography and Clinical Neurophysiology, 34, 818.

Thorne, J. (1965). Central responses to electrical activation of the peripheral nerves supplying the intrinsic hand muscle. Journal of Neurology, Neurosurgery, and Psychiatry, 28, 482-495.

Trontelj, J. V. (1973a). A study of the F-responses by single fibre electromyography. In New Developments in Electromyography and Clinical Neurophysiology. Edited by J. E. Desmedt, Volume 3, pp. 318-322. Karger: Basel.

Trontelj, J. V. (1973b). A study of the $\mathrm{H}$ reflex by single fibre EMG. Journal of Neurology, Neurosurgery, and Psychiatry. 36, 951-959.

Trontelj, J. V.. and Trontelj, M. (1973). F responses of human facial muscles. A single motor neurone study. Journal of the Neurological Sciences, 20, 211-222. 